\title{
Corela
}

Cognition, représentation, langage

7-1 2009

Vol. $7, \mathrm{n}^{\circ} 1$

\section{Pre- et post- en anglais contemporain : sémantisme et comportement accentuel}

\section{Véronique Abasq}

\section{OpenEdition}

\section{Journals}

Édition électronique

URL : http://journals.openedition.org/corela/145

DOI : $10.4000 /$ corela. 145

ISSN : 1638-573X

\section{Éditeur}

Cercle linguistique du Centre et de I'Ouest - CerLICO

Référence électronique

Véronique Abasq, «Pre- et post- en anglais contemporain : sémantisme et comportement accentuel», Corela [En ligne], 7-1 | 2009, mis en ligne le 26 juin 2009, consulté le 19 avril 2019. URL : http:// journals.openedition.org/corela/145; DOI : 10.4000/corela.145

Ce document a été généré automatiquement le 19 avril 2019

\section{(c) (i) (2)(2)}

Corela - cognition, représentation, langage est mis à disposition selon les termes de la licence Creative Commons Attribution - Pas d'Utilisation Commerciale - Partage dans les Mêmes Conditions 4.0 International. 


\title{
Pre- et post- en anglais contemporain : sémantisme et comportement accentuel
}

\author{
Véronique Abasq
}

\section{Introduction}

1 Ces dernières années ont vu paraître un nombre important de publications sur le sujet de la préfixation, tant en français qu'en anglais : un tel regain d'intérêt s'explique sans doute par le fait que les préfixes sont au cœur des interrogations sur le sémantisme morphologique, et dans le cas de l'anglais, notre sujet d'étude, sur leur rôle dans l'accentuation. Nous nous proposons ici d'explorer cette relation entre sémantisme des préfixes et accentuation à partir du cas particulier des préfixes pre- et post-, dont nous verrons qu'ils peuvent contribuer de façon tout à fait pertinente à en éclairer certains aspects. $^{1}$

\section{Les données}

\subsection{Corpus initial}

2 Le corpus initial est établi à partir des entrées principales des dictionnaires de prononciation Longman Pronunciation Dictionary 3rd edition, 2008 (J. C. Wells), ci-après LPD et Cambridge English Pronouncing Dictionary 17th edition, 2006 version 2.0 (D. Jones, P. Roach ed), ci-après EPD.

3 Ce corpus réunit toutes les entrées principales commençant par les séquences écrites <pre> et <post>. Les entrées secondaires, c'est-à-dire les entrées mentionnées au sein d'une entrée principale, ne sont pas prises en compte dans un premier temps : il s'agit pour l'essentiel de dérivés neutres. Le nombre d'entrées principales commençant par la 
séquence <pre> s'élève à 387 dans LPD et 316 dans EPD. Le nombre d'entrées principales commençant par la séquence <post> est de 77 dans LPD et 62 dans EPD :

- 111 entrées en <pre> de LPD ne sont pas attestées comme entrées principales dans EPD ;

- 40 entrées en <pre> de EPD ne sont pas attestées comme entrées principales dans LPD ;

- 20 entrées en <post> de LPD ne sont pas attestées comme entrées principales dans EPD ;

- 5 entrées en <post> de EPD ne sont pas attestées comme entrées principales dans LPD.

Le corpus initial comprend donc 427 entrées en <pre> et 82 en <post>.

\subsection{Des structures morphologiques différentes}

\subsubsection{Non préfixés}

\section{a) Cas évidents}

4 Une partie des entrées du corpus initial commençant par les séquences <pre> ou <post> ne sont pas historiquement préfixées en pre- ou post-. Sans avoir recours à l'étymologie, le statut de non préfixé de certaines entrées est plus que probable.

5 C'est le cas des noms propres. D'un point de vue synchronique aucun indice ne semble indiquer qu'ils pourraient être réinterprétés comme préfixés.

Preece, Preedy, Preminger, Prendergast, Prescelly, Prescot, Preshaw, Presley, Prestige, Prestatyn, Prestbury, Prestcold, Presteigne, Prestel, Prester, Preston, Prestonpans, Prestwich, Prestwick, Prestwood, Pret a Manger, Pretoria, Previn, Prewett

Posta, Postgate, Post-it, Postlethwaite, Postum

C'est aussi le cas d'entrées qui n'ont pas de structure morphologique interne apparente : preach, prey, prep...

6 Les mots formés à partir de press ne donneront pas non plus lieu à une interprétation préfixale de la séquence <pre> :

pressgang, pressie, pressing, pressman, pressmark, pressroom, press-stud, pressup, pressure, pressurization, pressurize...

7 La lexie post ne sera bien sûr pas décomposée. ${ }^{2}$ Il s'agit soit d'un substantif pouvant renvoyer à l'équivalent de « la poste » en français, à « un poste » dans le sens d'un emploi ou encore à « un poteau / montant », soit d'un verbe signifiant « poster », « annoncer ».

C'est à post dans le sens « la poste » que les entrées suivantes renvoient : postbag, postbox, postcard, post-chaise, postcode, post-free, post office, postmark, postman, postmaster, postmistress, postpaid, postage, postal, postie, posting

Dans ces exemples, post est soit premier élément de composition soit directement suffixé : il n'est en aucun cas interprétable comme préfixe. ${ }^{3}$

9 Les autres entrées qui ont post pour base, qu'il s'agisse du sens « emploi » ou autre, ne seront pas non plus réinterprétées comme préfixées : posture, poster, postern, postulate...

Enfin, il s'agit d'une préposition, et non d'un préfixe, dans le cas des expressions latines : post-bellum, post hoc, post meridiem, post-mortem, post partum

\section{b) Réinterprétation}

Les éléments écartés en a) n'entraînaient aucune confusion possible quant à leur statut. Parmi les entrées historiquement non préfixées restantes, on peut néanmoins se poser la question d'une réinterprétation préfixale synchronique. Un locuteur n'est en effet 
généralement pas conscient de l'étymologie des mots qu'il utilise; il établit dès lors, inconsciemment, la distinction entre les préfixés et les non préfixés en fonction notamment du sens et de la structure phonétique et morphologique. ${ }^{4}$

11 Certaines entrées de notre corpus présentent des agrégats consonantiques qui ne sont pas attestés en début de morphème, ce qui permet de conclure qu'elles ne peuvent pas être réinterprétées comme des préfixés : prednisone par exemple ne peut pas être décomposé en pre- + dnsisone puisque l'agrégat [dn] n'est pas attesté en début de morphème. La même analyse est pertinente pour prentice, presbytary, pretzel, où les agrégats [nt], [zb], [tz] ne sont pas non plus attestés en début de morphème.

Ces mêmes agrégats peuvent au contraire conduire à écarter des préfixés historiques comme dans pregnant, puisque l'agrégat [gn] n'est pas attesté en début de morphème.

En outre, dans le cas de pregnant l'interprétation suffixale l'emporterait probablement sur l'interprétation préfixale, -ant étant un suffixe adjectival largement répandu en anglais contemporain. Ce même argument peut être utilisé dans le cas de precatory ou encore pretty, entrées qui, elles, ne sont pas historiquement préfixées.

Du point de vue considéré ici, post-haste constitue un cas particulièrement intéressant. Il n'est historiquement pas préfixé : en effet, il s'agit en fait d'un composé de post dans le sens «la poste» et du substantif haste, la signification en étant ainsi originellement " aussi rapidement que par la poste ». Cependant la relation sémantique avec "la poste " est clairement perdue aujourd'hui. Or haste peut être la base de préfixés avérés : ainsi post-haste commute avec les mots forehaste, unhaste et over-haste. De plus, son schéma accentuel $/ 21 / 5$ est contradictoire avec la règle générale de placement de l'accent primaire des composés sur le premier élément de composition; au contraire, il s'apparente justement à celui des préfixés séparables.

De la même manière, nous serons conduits à reconsidérer l'analyse morphologique de posterior, posteriority et posterity, historiquement construits sur la préposition latine et non avec le préfixe. ${ }^{6}$

\section{c) Dérivés neutres}

Enfin, $L P D$ et $E P D$ incluent parmi leurs entrées principales un certain nombre de dérivés neutres, c'est-à-dire des dérivés dont l'accentuation et la prononciation ne diffèrent pas de celles du dérivant, et qui ne sont pas régis par une terminaison contraignante. Ainsi, les entrées preventative et preventable dérivent de prevent, et leurs suffixes -ative et -able, neutres, n'entraînent pas un recalcul de l'accent. Ces entrées seront elles-aussi exclues du corpus final. En revanche, l'entrée preventive dérive elle aussi de prevent, mais est régie par la terminaison contraignante $\mathrm{C}_{2}+$ suffixe adjectival en $-\mathrm{V}\left(\mathrm{C}_{0}(\mathrm{e})\right)^{7}$ imposant l'accent primaire sur la syllabe pénultième : cette entrée sera donc conservée. ${ }^{8}$

Quelques exemples:

postpositive < postpose

postpositional < postposition

preferment $<$ prefer

prepossessingly $<$ prepossessing $<$ prepossess

preservable $<$ preserve

prevaricator $<$ prevaricate

preventative/preventable $<$ prevent 
17 La construction des entrées suivantes est ambiguë : si elles sont construites sur le schéma de gauche, ce sont des dérivés neutres qui devraient donc être écartés du corpus :

\begin{tabular}{|l|l|}
\hline preamplifier : & preamplify +-er ou pre- + amplifier \\
\hline prepackage : & prepack +-age ou pre- + package \\
\hline prepayment : & prepay + -ment ou pre- + payment \\
\hline preshrunk : & preshrink + -ED ou pre- + shrunk \\
\hline
\end{tabular}

18 La potentialité même de la construction de droite nous a toutefois conduit à les conserver. Nous maintiendrons aussi le cas de preschooler pour des raisons d'interprétation sémantique que nous détaillerons en troisième partie, ce bien que la deuxième construction soit cette fois impossible, puisque ${ }^{\circ}$ schooler n'est pas attesté :

preschooler : preschool + -er ou pre- + ${ }^{\circ}$ schooler

\subsubsection{Préfixés}

\section{a) Origine}

19 Les préfixes pre- et post- sont d'origine latine. La forme initiale de pre- en latin était <prae>, elle devient <pre> en latin médiéval et c'est sous cette forme que le préfixe passe dans les langues romanes. Selon OED, on trouve encore la forme <prae> dans des mots anglais pour lesquels l'origine latine est clairement reconnue ou dans certaines formations néoclassiques.

20 En latin, pre- était principalement préfixé à des verbes ou à des déverbaux avec les sens : "before ", " in front ", " in advance ». L'emploi de pre- avec un adjectif ou un substantif dans ces sens était rare. Lorsqu'il était employé avec un adjectif, pre- était selon les termes de OED "an intensive to adjectives» comme par exemple dans praepotens: " exceedingly powerful ». Cet emploi du préfixe n'est pas productif en anglais contemporain.

21 Comme pre-, post- était lui aussi principalement préfixé à des verbes et à leurs dérivés dans les sens « after » et « behind».

22 De nombreux termes que l'on trouve aujourd'hui en anglais contemporain sont des emprunts au latin, directement ou par l'intermédiaire du français. A partir de ces emprunts, les préfixes se sont développés et sont restés des préfixes productifs pouvant en anglais contemporain être préfixés à des bases qui d'une part ne sont pas d'origine latine et d'autre part ne sont pas restreintes aux verbes ou déverbaux. Le préfixe pre- a par ailleurs supplanté le préfixe fore-.

\section{b) Le trait d'union}

L'utilisation du trait d'union varie d'un dictionnaire à l'autre. Selon OED, le trait d'union sera utilisé lorsqu'il s'agit d'une construction anglaise de type «nonce words " et "casual compounds» (constructions occasionnelles). Toujours selon OED, les mots directement 
empruntés au latin ou au français sont écrits en un seul mot, à l'exception des mots dont la base commence par un <e> comme pre-eminent ou pre-exist; ces derniers sont cependant écrits en un seul mot dans LPD. ${ }^{9}$ Pour les autres mots, l'usage du trait d'union est variable selon les auteurs.

\section{c) Plusieurs types de préfixés}

L'évolution de ces préfixes fait qu'en anglais contemporain trois types de constructions sont observables :

- Construction séparable avec base libre : prepay, precook, prehistory, prestressed, postwar...

- Construction inséparable avec base libre : predominant, premeditate, prepossess...

- Construction inséparable avec base liée : precise, prefect, postpone...

Nous reviendrons plus en détails sur cet aspect dans la partie 3 ci-après.

\section{Accentuation et prononciation}

\subsection{Schémas généraux d'accentuation des préfixés}

\subsubsection{Préfixés séparables}

La base des préfixés séparables reçoit un accent primaire, dont le placement obéit aux règles générales d'accentuation de l'anglais. Le préfixe est lui aussi accentué. L. Guierre (1979) lui attribuait un accent primaire sur la base des transcriptions de la $12^{\text {ème édition }}$ de l'EPD ; à partir de la $13^{\text {ème }}$ édition, cet accent est désormais secondaire. LPD adopte le même principe.

Le schéma général des préfixés séparables est donc : $/ 2 /+/ 1 /$, quelle que soit la catégorie grammaticale du préfixé.

\subsubsection{Préfixés inséparables}

L'accentuation des préfixés inséparables dépend en grande partie de la catégorie grammaticale à laquelle ils appartiennent: les préfixés monocatégoriels non substantifs (c'est à dire les verbes, les adverbes, les adjectifs) reçoivent l'accent primaire sur la base ; cependant la présence d'une terminaison contraignante primera sur la catégorie quitte à ce que l'accent soit placé sur le préfixe comme dans 'previous

Le préfixe lui-même est inaccentué. S'il reçoit un accent secondaire, celui-ci est dû aux conséquences du troisième principe d'accentuation qui interdit de commencer un mot par deux syllabes inaccentuées.

29 Les préfixés substantifs monocatégoriels suivent les schémas généraux d'accentuation :

- dissyllabes en /10/

- $C_{2}$ préfinal, mots « italiens $»^{10}$ en /-10/

- trisyllabes et plus en /(-)100/

La présence du préfixe n'implique pas de comportement accentuel particulier.

Les préfixés pluricatégoriels substantif/verbe suivent soit le schéma des verbes (accentuation sur la base), soit le schéma des substantifs (accentuation sur le mot entier), soit leur schéma respectif. 

substantifs. ${ }^{11}$

\subsection{Analyse du corpus}

Selon EPD, dans les mots où la base est libre et où pre- signifie "beforehand", pre- reçoit généralement un accent secondaire. Selon LPD, s'il s'agit du préfixe productif signifiant « beforehand ", le préfixe reçoit un accent, qu'il soit primaire ou secondaire.

Seul LPD fait un commentaire sur le préfixe post- mais celui-ci ne concerne pas l'accentuation.

\subsubsection{Séparables}

Les entrées a priori séparables de notre corpus sont les suivantes :

post- :

postconsonantal, postdate, post-diluvian, postdoc, postdoctoral, postgame, postglacial, postgraduate, posthypnotic, postimpressionism, postimpressionist, postindustrial, postmenopausal, post-modern, postnasal, postnatal, postnuptial, post-op, postproduction, postseason, postsecondary, poststructural, posttraumatic, postvocalic, postwar, postviral.

pre- :

preadamic, preadamite, preamplification, preamplify, prearrange, prebuilt, ${ }^{12}$ precambrian, precancerous, precast, preclassical, preclinical, pre-colonial, preColumbian, precompose, preconceive, preconcert, precondition, preconsonantal, precook, predate, ${ }^{13}$ predawn, predecease, predefine, predetermination, predetermine, predigest, pre-echo, pre-eclampsia, preexilian, pre-exilic, preexist, preexistence, preexistent, prefabricate, prefabrication, preflight, preformation, prefrontal, pregame, preheat, prehistoric, prehistory, pre-ignition, prelapsarian, prelingual, preliterate, pre-loved, premarital, premed, premedical, premedication, premenstrual, premodification, premodify, premolar, prenatal, prenuptial, pre-op, preoperative, preordain, preordination, pre-owned, prepack, prepackage, prepaid, prepay, preproduction, preprogrammed, Pre-Raphaelite, prerecord, preregister, prerequisite, preschool (adj), prescientific, preseason, preset, preshrink, preshrunk, pre-Socratic, presort, ${ }^{14}$ prestressed, pretax, preteen, preterm, pretonic, pretrial, preverbal, prevocalic, prewar, prewash

Conformément aux règles énoncées plus haut, le préfixe reçoit un accent secondaire et la base l'accent primaire.

Dans tous ces cas, le préfixe n'entre pas en ligne de compte pour le calcul de l'accent primaire : la base libre est accentuée comme lorsqu'elle est autonome, ce qui fait que dans quelques cas deux accents secondaires sont attestés :

,pre-,amplifi'cation, ,pre,conso'nantal, ,post,conso'nantal, ,prede,termi'nation, , pre,fabri'cation, ,pre,modifi'cation, ,pre,scien'tific.

Aux entrées ci-dessus s'ajoutent les 9 suivantes qui ne suivent pas ce comportement accentuel et reçoivent l'accent primaire sur le préfixe :

'pre-amp, 'prehead, 'preprint, 'pretest, 'posttest ${ }^{15}$

'postgrad, ${ }^{16}$ 'preschool (n), ${ }^{17}$ 'prefab.

39

On notera qu'à l'exception de ,pre'teen, il s'agit de la totalité des substantifs préfixés séparables dissyllabiques, soit une caractéristique potentiellement déterminante. 


\subsubsection{Inséparables}

post-

43 notre corpus. On en décompte en tout et pour tout 7 soit $25 \%$, proportion qui chute considérablement si l'on intègre le grand nombre d'entrées séparables identifiées par OED :

postpone, postlude, postpose, postposition, postfix, postscript, posthumous. ${ }^{18}$

44 'postlude est un substantif dans LPD. Il suit le schéma général des substantifs préfixés inséparables : il s'agit d'un dissyllabe, il est donc accentuée en /10/. La même analyse s'applique d'ailleurs à 'prelude. ${ }^{19}$

Il en va de même pour les substantifs 'postfix et 'postscript même si la frontière entre séparable et inséparable n'est pas aussi claire que dans postlude dans ces cas. L'existence de prefix notamment nous amènera à reconsidérer ce type d'entrées. ${ }^{20}$ post' pone est un verbe préfixé monocatégoriel inséparable, il reçoit donc l'accent primaire sur la base. Il s'agit de la seule entrée pour laquelle le $<0>$ de $<$ post> peut être prononcé [ə].

47 ,post'pose est comme post' pone un verbe préfixé monocatégoriel inséparable, il reçoit donc lui aussi l'accent primaire sur la base. Cependant, l'accent secondaire noté par LPD semble aller à l'encontre des règles d'accentuation des préfixés inséparables. Le verbe ,pre' pose ${ }^{21} \mathrm{a}$ le même schéma accentuel. Tout comme postfix dont la base est une base libre, il faudra revenir sur le degré d'inséparabilité de ces entrées.

,postpo'sition est contraint par la terminaison en $-i+V\left(C_{0}(e)\right)$. On devrait donc considérer ici que l'accent secondaire placé sur le préfixe est dû à une contrainte rythmique (un mot ne peut pas commencer par deux syllabes inaccentuées). 'posthumous est un adjectif monocatégoriel préfixé inséparable. Il devrait recevoir l'accent primaire sur la base. Ives Trevian avance que dans les séquences VCous, -ous contraint l'accent sur la syllabe antépénultième ${ }^{22}$ : posthumous ne serait alors pas perçu comme un préfixé. C'est d'ailleurs la seule entrée historiquement préfixée pour laquelle la voyelle de post n'est pas la diphtongue [əひ] mais la voyelle brève [0] que l'on trouve dans certaines entrées historiquement non préfixées telles: posture, postern, Postum... Dans cette hypothèse, la totalité de ces cas serait donc conforme aux règles d'accentuation.

pre- 
Le nombre d'entrées inséparables en pre- est sans commune mesure avec le nombre d'entrées en post-. De manière générale elles obéissent aux règles générales d'accentuation :

- Terminaisons contraignantes : ,predica'bility, ,predi'cation, 'predial, pre'ponderate...

- Préfixés non substantifs : pre'cede, pre'dict, pre'fer, pre'pare, pre'posterous...

- Dissyllabes 盛/10/ : 'precept, 'prefect, 'prelate, 'pretext...

- $C_{2}$ préfinal 閶/-10/: pre'centor, pre'ceptor

- (Mots « italiens » : aucun)

- Règle normale 䶊/(-)100/ : prejudice, pre'rogative...

\subsubsection{Pluricatégoriels}

Le tableau ci-dessous regroupe les pluricatégoriels explicitement identifiés comme tels par LPD et EPD. Comme souvent dans le cas des pluricatégoriels, le nombre de variantes est élevé et les dictionnaires souvent divergents. Tous les substantifs de ce corpus reçoivent néanmoins l'accent primaire sur le préfixe, en raison de la terminaison contraignante -ent dans precedent et conformément à la règle des dissyllabes pour les autres.

\begin{tabular}{|c|c|c|c|c|c|c|}
\hline \multirow[t]{2}{*}{ prefixé } & \multicolumn{3}{|l|}{$L P D$} & \multicolumn{3}{|l|}{$E P D$} \\
\hline & substantif & verbe & adjectif & substantif & verbe & adjectif \\
\hline precedent & $/ 100 /$ & & $\begin{array}{l}/ 010 / \\
(/ 100 /)\end{array}$ & $/ 100 /$ & & $\begin{array}{l}/ 010 / \\
(/ 100 /)\end{array}$ \\
\hline preface & $/ 10 /$ & $/ 10 /$ & & & & \\
\hline prefix & $/ 10 /$ & $\begin{array}{l}/ 10 / \quad(/ \\
(2) 1 /)\end{array}$ & & $/ 10 /$ & $\begin{array}{l}/ 21 / \\
(/ 10 /)\end{array}$ & \\
\hline premise & $/ 10 /$ & $\begin{array}{l}/ 10 / \\
(/ 01 /)\end{array}$ & & $/ 10 /$ & $\begin{array}{l}/ 01 / \\
(/ 10 /)\end{array}$ & \\
\hline presage & $/ 10 /(/ 01 /)$ & $\begin{array}{l}/ 10 / \\
(/ 01 /)\end{array}$ & & $/ 10 /$ & $\begin{array}{l}/ 10 / \\
(/ 01 /)\end{array}$ & \\
\hline prescript & $/ 10 /$ & & $/ 01 /$ & & & \\
\hline present & $\begin{array}{l}/ 10 / \text { mais /01/ au } \\
\text { sens de "military } \\
\text { stance" }\end{array}$ & /01/ & $/ 10 /$ & $\begin{array}{l}/ 10 / \text { mais /01/ au } \\
\text { sens de «military } \\
\text { term» }\end{array}$ & /01/ & $/ 10 /$ \\
\hline
\end{tabular}

\subsubsection{Dérivés exceptionnels}

Parmi les dérivés à suffixe neutre 3 entrées sont exceptionnelles :

pre'dicative < 'predicate

'preferable < pre'fer 
Dans ce cas, Jean-Louis Duchet (1988) fait l'hypothèse que -able devient contraignant lorsqu'il a le sens « qui doit être » plutôt que son sens habituel « qui peut être ». pre'fectural ${ }^{23}<$ 'prefecture

Bien qu'il n'y ait que très peu de cas pertinents, -ural pourrait être considérée comme une terminaison contraignante.

On relève aussi deux exceptions à terminaison contraignante en -ent de plus de 2 syllabes $\rightarrow /(-) 100 /:$ pre'potent /010/, pre'cedent (adj)

On note enfin le cas de preamble dont l'accentuation est exceptionnelle à la règle des dissyllabes en variante principale selon LPD. EPD répertorie les variantes en sens inverse :

LPD pre'amble variante 'pre,amble

EPD 'preamble variante pre'amble

\subsubsection{Accent secondaire}

LPD et EPD notent quelques entrées inséparables pour lesquelles l'accent primaire est immédiatement précédé d'un accent secondaire sur le préfixe, en contradiction avec les principes rythmiques d'accentuation du mot en anglais :

,pre'destinate, ,pre'buttal, ,pre-'empt, ,post'pose...

Comme les préfixés séparables dont l'accent primaire est sur le préfixe, ${ }^{24}$ ces quelques cas a priori exceptionnels renvoient à la question de l'interprétation sémantique qui fait l'objet de la partie suivante. Pour le reste, les préfixés en pre- ou en post- sont réguliers et obéissent pour le plus grand nombre aux règles générales de l'accentuation de l'anglais.

\section{Sémantisme : séparable ou inséparable?}

Les concepts de séparabilité et d'inséparabilité sont notamment d'ordre sémantique. ${ }^{25}$ Comme nous l'avons vu en 1.2.2. c), on observe 3 types de constructions :

- Construction séparable avec base libre : prepay, precook, prehistory, prestressed, postwar...

61 Le préfixe est porteur d'une signification propre et indépendante de celle de la base. Il est susceptible d'être perçu comme distinct du reste du mot et son contenu sémantique est défini. La base est une unité lexicale. Le sens général de la construction se déduit du sens du préfixe et du sens courant de la base. ${ }^{26}$

2 On peut distinguer 2 types de constructions inséparables :

- Construction inséparable avec base libre : predominant, premeditate, prepossess...

- Construction inséparable avec base liée : precise, prefect, postpone..

Dans ces constructions, de manière générale, le sens du préfixé ne peut pas être directement déduit du sens du préfixe et du sens de la base. On notera cependant que dans les cas où le préfixe est suivi d'une base libre, la non reconstruction du sens ne va pas de soi. Lorsque la base est liée, ${ }^{27}$ il faudra néanmoins revenir sur le sémantisme : si la construction est inséparable, le préfixe ne véhicule-t-il tout de même pas du sens? 


\subsection{Constructions séparables}

64 Nous venons de le voir, dans les constructions séparables, le sens se déduit directement $d u$ sens du préfixe et du sens courant de la base. Les dictionnaires indiquent ainsi le sens «beforehand" pour pre- et le sens «after» pour post-. Cependant dire que pre- signifie «avant » et post- " après » ne rend pas suffisamment compte des deux «valeurs ", ou "rôles", sémantiques distinctes et des variations de sens que les préfixes peuvent exprimer.

\subsubsection{Les deux « valeurs » sémantiques}

OED distingue deux valeurs de base pour les préfixes pre- et post- :
«A. pre- is adverbial or adjectival, qualifying the verb, adjective or substantive to which it is prefixed."
«B. Combinations in which pre- is prepositional, the object being the noun forming, (or implied in), the second element. »
"A. post- is adverbial or adjectival, qualifying the verb, verbal derivative, or other adjectives or nouns forming or implied in the second element."
«B. Compounds in which post- is prepositional, the object being the noun forming, or
implied in the second element. $\|^{28}$

Cette distinction entre valeur adjectivale ou adverbiale et valeur prépositionnelle du préfixe rejoint la distinction entre préfixés endocentriques et préfixés exocentriques reprise par D. Amiot (2003) à l'occasion de l'analyse des substantifs en pré- et post-en français. ${ }^{29}$ Elle explique ainsi que dans le cas de "prérapport» il s'agit bien d'un « rapport », et qu'on peut dès lors qualifier le préfixé d'endocentrique puisqu'il n'y a pas besoin d'un élément extérieur pour reconstruire son sens, ce qui rejoint la valeur adjectivale ou adverbiale du préfixe selon OED. Dans le cas de «l'après-guerre » au contraire, il ne s'agit pas d'une guerre: c'est cette fois la valeur prépositionnelle du préfixe qui est en jeu; le préfixé est alors exocentrique. Comme elle l'indique, ces termes d'endocentrique et exocentrique sont originellement dûs à L. Bloomfield dans le domaine de la syntaxe, puis adaptés à la morphologie par E. Benveniste et A. Martinet.

Cette différence de fonctionnement se double en outre d'une différence portant cette fois sur le sémantisme des préfixes et non plus sur les constructions auxquelles ils participent, c'est-à-dire entre leur emploi temporel et leur emploi locatif, auxquels s'ajoute un emploi intensif de pre-sur lequel nous reviendrons en fin de cette partie. Comme on va le voir, l'emploi temporel peut lui-même faire l'objet de distinctions complémentaires.

\subsubsection{Emploi locatif}

pre- et post- peuvent en effet être locatifs :

prefrontal, prelingual, ${ }^{30}$ premolar, postnasal

toutefois le nombre de cas concernés est peu élevé et cet usage est limité pour l'essentiel aux domaines connexes de la médecine, de l'anatomie et de la zoologie. Contrairement au comportement fréquent des adverbes, à la fois temporels et spatiaux, ces deux préfixes apparaissent ainsi particulièrement spécialisés dans le domaine temporel. 


\subsubsection{Emploi temporel}

\section{a) Valeur adverbiale ou adjectivale du préfixe}

- « avant » / « à l'avance ", « après » :

precook, preamplify, preamplification, pre-echo, prearrange, prestressed, postdate, postproduction...

- « avant » / « déjà »:

pre-loved, pre-owned

\section{b) Valeur prépositionnelle du préfixe (pas de verbes)}


différent. Néanmoins, les dictionnaires ne notent pas de différence d'accentuation entre les deux acceptions alors que le substantif est accentué /10/ et l'adjectif /21/.

\subsubsection{Observations complémentaires sur l'aspect sémantique}

Nous conclurons cette partie sur quelques cas particuliers qui illustrent d'autres aspects de cette problématique. Le premier d'entre eux, predecease constitue d'un certain point de vue une extension du phénomène observé avec pre-loved et pre-owned, plus précisément une spécialisation de la valeur temporelle due aux caractéristiques référentielles de la base. En effet, predecease ne peut pas signifier « décéder à l'avance ». Le sens attesté dans les dictionnaires est en fait " décéder avant quelqu'un d'autre »: l'antériorité porte alors sur le complément d'objet (le verbe est un verbe transitif).

Le deuxième cas de figure concerne le rapport entre troncation et séparabilité. Les exemples suivants :

'pre-amp, 'prefab, 'postgrad, ,pre-'med,,post-'op

sont en effet issus de :

,pre-'amplifier, ,pre'fabricated, ,post'graduate, ,pre-'medical, ,post-'operative.

81 Comme on peut le constater, les trois premiers ont un schéma accentuel différent des mots dont ils sont issus. Comme on l'observait dans la deuxième partie, ce comportement ne peut pas être attribué au seul phénomène de la troncation puisqu'il n'affecte pas premed et post-op. De façon générale la question que pose le phénomène de la troncation est celle du maintien de l'identité sémantique en dépit de la modification de la forme. Dans ces cas s'ajoutent la perte du déterminant accentuel que constitue la terminaison contraignante, et l'attraction vers le modèle des substantifs dissyllabiques préfixés dont on a observé qu'ils sont accentués /10/ même lorsqu'ils sont séparables. C'est peut-être ce dernier paramètre qui explique la différence de comportement puisque pre-med et pre-op sont des adjectifs (avec un emploi nominal par conversion dans le cas de pre-med: « $a$ premedical student»), tandis que les trois premiers sont des substantifs; prefab est d'ailleurs un substantif alors que prefabricated est un adjectif.

Enfin, l'emploi intensif. Le préfixe pre- découlant du préfixe latin prae- peut aussi exprimer la supériorité. ${ }^{31}$ C'est le cas par exemple de :

prepotent, predominant, pre-eminent...

Cependant cet usage du préfixe est a priori non productif en anglais contemporain : on pourrait dès lors penser que cette caractéristique rend douteuse l'analyse de ces constructions comme séparables. Cependant, l'existence de paires commutantes telles predominant $\sim$ co-dominant $\sim$ subdominant ou encore pre-eminent $\sim$ supereminent, suggère pour le moins un certain degré de pertinence dans l'interprétation du préfixe. Nous verrons plus loin que ces réseaux de relations morpho/sémantiques jouent sans aucun doute un rôle dans cette perspective.

\subsection{Constructions inséparables : degrés d'inséparabilité}

\subsection{1. postfix, postlude, prebuttal, prequel}

On pourrait admettre que le verbe prefix soit perçu comme séparable. Ce n'est pas le cas du substantif, notamment en raison de sa limitation aux domaines de la morphologie et de la téléphonie. Cependant, c'est bien sur le modèle du substantif que postfix a été créé, 
en exploitant la différence sémantique entre post- et pre- ce qui implique que celui-ci restait interprétable malgré l'inséparabilité du substantif prefix.

On observe le même phénomène dans le cas de postlude, formé par analogie avec prelude et interlude, sans que lude, base liée d'origine latine, ne soit cette fois interprétable. Néanmoins les valeurs temporelles de pre-, inter- et post- restent transparentes dans les préfixés : post- étant le préfixe le plus productif permettant d'exprimer la postériorité, il est utilisé.

'buttal, le néologisme n'est pas formé sur une interprétation temporelle du préfixe re- mais sur la perception du mot tout entier: a re'buttal se fait en réponse à quelque chose, donc «après ». À son tour, pre- étant le préfixe le plus productif permettant d'exprimer l'antériorité temporelle, il est utilisé. L'accent secondaire de „pre' buttal, précisément absent de re'buttal, marque ce contraste (Rappelons qu'il ne peut y avoir deux accents successifs au sein d'un même mot).

Notre dernier exemple est prequel. Comme pour rebuttal, a sequel est quelque chose qui « vient après ». Comme <buttal>, <quel> n'est pas une base mais contrairement à rebuttal, sequel n'a pas de préfixe. ${ }^{32}$ Et pourtant, il s'agit bien du même phénomène sémantique.

De ce point de vue, si posterior, posteriority et posterity ne sont pas historiquement préfixés, puisque post y représente la préposition latine, on peut faire l'hypothèse d'une réinterprétation préfixale, tant pour des raisons de sens qu'à cause de la commutation avec exterior.

89 Les cas examinés dans cette partie témoignent d'un réinvestissement du sens; au contraire, dans la partie suivante nous nous intéresserons à la persistance du sens en dépit de l'opacification.

\subsubsection{Opacification}

En effet, si les exemples suivants témoignent d'une certaine opacification malgré leur base libre, ce qui conduit à les traiter comme inséparables :

prevision, prescience, ${ }^{33}$ precognition, predispose, presentiment, predestination...

91 opacification d'ailleurs confirmée par leur comportement phonétique comme le fait remarquer Susan Moore-Mauroux (2008) à propos du changement de prononciation du $<\mathrm{s}>$ de sentiment, ${ }^{34}$ ils préservent cependant clairement le sens du préfixe.

Même dans le cas de constructions telles pre-empt et prevent, le sens temporel du préfixe a persisté au-delà de l'opacité complète de la base. D'ailleurs, si prevent au sens « hinder » est accentué /01/, il prend l'accentuation /21/, tout à fait exceptionnelle, dans son sens le plus clairement temporel de «go before » selon EPD.

\subsubsection{Quelques cas complémentaires}

\section{preconceive}

93 Nous voudrions ici ajouter que si certains suffixes lexicaux sont neutres d'un point de vue accentuel, il faut les réintroduire lorsque l'on s'intéresse au sémantisme. Les suffixes qui traditionnellement sont décrits comme changeant la catégorie grammaticale peuvent-ils entraîner une réinterprétation du préfixé? Si preconceive peut s'interpréter comme « concevoir à l'avance » sans jugement moral, il n'en va pas de même pour preconception 
et preconceived qui dans leur acception la plus courante véhiculent une valeur moralement négative.

premature

donne un preference poll en anglais britannique : $59 \%$ des locuteurs l'accentuent en /100/ et $41 \%$ en /201/. LPD n'explique pas cette disparité cependant nous pouvons faire l'hypothèse d'une différence de perception sur sa séparabilité. Si le sens d'un préfixé séparable peut se déduire du sens du préfixe et du sens de la base, ceci n'implique pas que le locuteur qui l'utilise le fasse.

\section{posthumous}

Si nous partageons l'interprétation inséparable de posthumous que propose Susan MooreMauroux,,$^{35}$ il paraît cependant difficile de contester la pertinence de la valeur temporelle dans ce mot, et donc d'une forme de reconnaissance du préfixe a priori réfutée par la nature entravée de la voyelle. La solution à ce paradoxe pourrait se trouver dans le suffixe : s'il est en effet contraignant comme le suppose I. Trevian, ${ }^{36}$ il impose alors d'un point de vue phonologique le recalcul de la voyelle, et donc son entravement, indépendamment de la reconnaissance ou non du préfixe en tant que tel.

\section{Conclusion}

Cette exploration du comportement sémantique des constructions en pre- et post-, du point de vue de la morphophonologie, met en évidence un aspect de la relation entre accentuation et sémantisme que nous avions abordé lors de l'étude des constructions en over-, under-, out-, up- et down-. ${ }^{37}$ Si l'opposition entre séparabilité et inséparabilité rend compte d'un nombre important de cas, les exemples que nous avons étudiés démontrent que l'inséparabilité sémantique ne signifie pas qu'aucun trait sémantique ne puisse être perçu. Or, on a pu constater que le sens propre du suffixe peut suffire à générer des accentuations a priori contradictoires avec les comportements accentuels établis par Lionel Guierre. Il nous semble qu'un tel phénomène est directement lié à l'importance que le locuteur accorde à cette information, comme l'illustre en particulier le cas de premature.

97 Cette question ne saurait bien sûr être résolue à partir de l'étude de ces deux seuls préfixes même s'ils apparaissent particulièrement intéressants de ce point de vue. D'autres recherches devraient permettre d'en évaluer la pertinence, mais sans doute aussi l'élargissement $d u$ corpus de référence: la simple consultation $d u$ web fait apparaître un nombre considérable de préfixés en pre- et post- absents d' EPD et LPD ; sans aucun doute, les unités répertoriées par ces dictionnaires ont-elles aussi d'autres acceptions, et d'autres accentuations, que celles mentionnées. 


\section{BIBLIOGRAPHIE}

Abasq V (2007), Préfixation et particules adverbiales en anglais contemporain : étude du comportement accentuel, Thèse de doctorat : Université François-Rabelais de Tours.

Amiot D.(2003), « De l'antériorité à la postériorité », Modes de repérages temporels, Cahiers Chronos $n^{\circ} 11, \mathrm{~S}$. Mellet et M. Vuillaume éd., Amsterdam/New York : Rodopi.

Bloomfield L. (1933), Language, traduction française (1970), Paris : Payot.

Deschamps A., Duchet J.-L. Fournier J.-M. et O’Neil M. (2004), English Phonology and Graphophonemics, Paris : Ophrys.

Duchet J.-L. et Fournier J.-M. (1988), « Isomorphisme et productivité dans l'accentuation et la prononciation des mots dérivés anglais ", $4^{\mathrm{e}}$ colloque d'avril sur l'anglais oral, P. Larreya \& J. Humbley, éd., Université de Paris-Nord : CELDA, diffusion APLV

Duchet J.-L. (1991), Code de l'anglais oral, Gap : Ophrys.

Fournier J.-M. (1996), « La reconnaissance morphologique », $8^{\circ}$ colloque d'avril sur l'anglais oral, J.-L. Duchet, J.-M. Fournier J. Humbley \& P. Larreya, éd., Université de Paris-Nord : CELDA, diffusion APLV.

Guierre L. (1979), Essai sur l'accentuation en anglais contemporain - Eléments pour une synthèse, Paris, Université Paris-VII.

Guierre L. (1984), Drills English Stress-Patterns, 4th ed., Londres : A. Colin - Longman.

Guierre L. (1990), « Conflits internes et externes en phonétique de l'anglais », 5e colloque d'avril sur l'anglais oral, J.-L. Duchet, J.-M. Fournier, J. Humbley \& P. Larreya, éd. , Université de ParisNord : CELDA, diffusion APLV.

Moore-Mauroux S. (2008), « Etre ou ne pas être un préfixe », Préfixation, prépositions, postpositions : études de cas, M. Paillard dir., collection Rivages Linguistiques, Rennes : P.U.R. Trevian I. (2003), Morphoaccentologie et processus d'affixation de l'anglais, Bern : Peter Lang.

\section{Dictionnaires}

Jones, D. (2006), Cambridge English Pronouncing Dictionary, 17th edition, edited by P. Roach, J. Setter \& J. Hartman, Cambridge : CUP.

Oxford English Dictionary, 2nd ed. Vers. 3.00 CD-ROM. (2002), Oxford : Oxford UP.

Wells, J.C. (2008), Pronunciation Dictionary, 3rd edition, London : Longman.

\section{NOTES}

1. Sur le même sujet, on lira notamment l'étude de Susan Moore-Mauroux sur pre-, post-, ex- et fore-, avec une perspective et des objectifs complémentaires de ceux retenus ici ; qu'elle les ait 
inclus dans sa liste confirme que ces deux préfixes constituent sans aucun doute un terrain particulièrement fertile pour ce champs de réflexion.

2. pre- quant à lui ne peut pas apparaître seul. L'Oxford English Dictionary (ci-après OED) donne un usage de pre en tant que préposition, dans deux citations seulement, en précisant qu'il s'agit d'une extension du préfixe pre- par analogie avec post prépositionnel, et qu'il est « usu. found in contexts where before would be equally appropriate and more agreeable » :

1973 G. Sims Hunters Point xiii 119 « Have you tried phoning David's friends in Los Angeles? ».. "They are all pre my era and I don't know their names. "

1975 H. Kissinger in Dept. of State Bull. 6 Oct. 532 «Pre my being in office; my decisions were made in the previous Administration. "

3. A l'exception de ,post-'free et ,post-'paid, ils reçoivent l'accent primaire sur "post». Les dictionnaires notent un accent secondaire lorsque le deuxième élément comprend plus d'une syllabe. L'accent secondaire post tonique représente la non réduction de la voyelle du second élément de composition. La voyelle du deuxième élément n'est pas réduite sauf dans le cas de postman. L'évolution de -man vers une interprétation suffixale explique sans doute cette réduction.

4. Voir en particulier Lionel Guierre (1990) et Jean-Michel Fournier (1996).

5. Où $/ 2$ / représente l'accent secondaire et /1/ l'accent principal.

6. Voir 3.2.1. ci-après.

7. Où $C_{2}$ représente un agrégat consonantique, et $C_{0}$ un nombre quelconque de consonnes.

8. En effet, si le caractère contraignant est établi pour les suffixes adjectivaux monosyllabiques, et donc -ive, il n'en va pas de même des suffixes adjectivaux dissyllabiques et en particulier de ative : ainsi, la liste proposée par I. Trevian (2003, p. 109) comprend en fait essentiellement des cas non-pertinents, justement contredits par les cas pertinents exceptionnels.

9. Jean-Louis Duchet fait remarquer à ce propos la sous-utilisation du trait d'union dans LPD (communication personnelle).

10. Mots terminés par une consonne alvéolaire différente de $<r, l>$ suivie d'une voyelle simple différente de $<\mathrm{y}>$ : to'mato, mar'tini ...

11. Pour ce comportement des préfixés non substantifs, voir L. Guierre (1984), J.-L. Duchet (1991),

A. Deschamps, J.-L. Duchet, J.-M. Fournier et M. O'Neil (2004).

12. Uniquement dans $E P D$.

13. $L P D$ précise dans le sens " antedate », donc pas celui de " prey on ».

14. Uniquement dans EPD.

15. Attestés uniquement dans LPD.

16. Avec une variante en $/ 21$ / dans LPD mais aucune variante pour EPD.

17. Dans LPD, preschool est pluricatégoriel substantif/adjectif, le substantif est accentué en /10/ et l'adjectif en /21/. Dans $E P D$, aucune catégorie n'est indiquée. La première accentuation donnée est $/ 10 /$ avec une variante en $/ 21 /$.

18. postfix, postlude et postpose ne sont pas attestés dans EPD.

19. Selon OED, postlude peut aussi être un verbe accentué en /10/, découlant du substantif, et qui en garderait donc l'accentuation. Il en va de même du verbe prelude, accentué /10/ depuis le milieu du $19^{\text {ème }}$ siècle sur le modèle du nom ("after the noun »).

20. OED atteste un substantif et un verbe postfix. Le verbe est accentué en /01/. Il s'agit d'une construction par analogie avec prefix.

21. Non attesté dans EPD.

22. I. Trevian (2003), pp 120-121.

23. Attesté uniquement dans $L P D$ avec une variante régulière en $/ 1000 /$.

24. Voir 2.2.1. ci-dessus.

25. Voir en particulier le texte fondateur de L. Bloomfield (1933) sur cette question, notamment pp 168-173 et chapitre XIV, pp 213-230, de la traduction française. 
26. Selon L. Guierre (1979), p. 358.

27. Dans notre corpus, il s'agit de racines latines pour la plupart.

28. On notera l'utilisation du terme «combinations» lorsqu'il s'agit du préfixe pre- et de " compounds » lorsqu'il s'agit de post-, ce qui implique une différenciation que OED n'explicite pas.

29. p. 183.

30. prelingual est séparable lorsqu'il est locatif ( located in front of the tongue »), mais inséparable lorsqu'il est temporel (" antecedent to the development or acquirement of language »)

31. Voir 1.2.2., a).

32. En raison de ce processus, «quel » pourrait sans doute devenir à son tour une base signifiante.

33. Que l'on comparera à prescientific.

34. p. 69.

35. p. 70.

36. Voir 2.2.2.

37. V. Abasq (2007)

\section{RÉSUMÉS}

Cet article vise à vérifier l'hypothèse d'une corrélation entre sémantisme et schéma accentuel à partir de l'étude de deux préfixes appartenant au domaine spatial : pre- et post-. L'opposition entre préfixé sémantiquement séparable et préfixé sémantiquement inséparable rend compte de l'accentuation d'un nombre important de cas, cependant, le fait qu'un préfixé ne soit pas séparable ne signifie nullement qu'aucun trait sémantique ne puisse être perçu et la réinterprétation morphologique à partir du sémantisme peut générer des schéma accentuels différents comme l'atteste le cas de premature par exemple. L'inverse peut aussi se produire dans le cas d'une unité sémantiquement séparable comme prehistory, où il se peut que le locuteur n'interprète pas la construction et l'accentue indépendamment de ses éléments constituants.

In this paper we explore the possible correlation between stress pattern and semantics based on the study of two prefixes with spatial meaning: pre- and post-. The opposition between separable and inseparable prefixed words explains the different stress patterns for most cases. However, even when the prefix is semantically inseparable, some meaning can be perceived and the reinterpretation of the prefixed word can lead to variants of the stress pattern as seems to be the case with premature. On the other hand, separable units such as prehistory may well be interpreted and stressed independently from their two constituting elements.

\section{INDEX}

Keywords : morphology, semantics, stress patterns, english

Mots-clés : morphologie, sémantisme, accentuation, préfixation, anglais 


\section{AUTEUR}

\section{VÉRONIQUE ABASQ}

Université François Rabelais Tours, Laboratoire Ligérien de Linguistique 